\title{
Alteration of melatonin secretion in patients with type 2 diabetes and proliferative diabetic retinopathy
}

This article was published in the following Dove Press journal:

Clinical Ophthalmology

I8 May 201 I

Number of times this article has been viewed

\author{
Taiichi Hikichi' \\ Naohiro Tateda ${ }^{2}$ \\ Toshiaki Miura ${ }^{3}$ \\ 'Department of Ophthalmology, \\ Ohtsuka Eye Hospital, Sapporo; \\ ${ }^{2}$ Asahikawa National College of \\ Technology, Asahikawa; ${ }^{3}$ Faculty of \\ Pharmaceutical Sciences, Hokkaido \\ University, Sapporo, Japan
}

Correspondence: Taiichi Hikichi Ohtsuka Eye Hospital, Kita-I6 Nishi-4, Kita-ku, Sapporo 00 I-00 I6, Japan Tel +8 I I | 74752 I I

Fax +8I II 7575223

Email taiichi-hikichi@hokkaido.med.or.jp
Background: The purpose of this study was to evaluate the dynamics of plasma melatonin secretion in patients with type 2 diabetes mellitus and diabetic retinopathy.

Methods: Plasma melatonin levels were measured by high-performance liquid chromatography in 56 patients. Patients were divided into a diabetic group (30 patients) and a nondiabetic group (26 patients). The diabetic group was divided further into a proliferative diabetic retinopathy (PDR) group $(\mathrm{n}=14)$ and a nonproliferative diabetic retinopathy (NPDR) group $(\mathrm{n}=16)$. Plasma melatonin levels obtained at midnight and 3 am were compared between the groups.

Results: Nighttime melatonin levels were significantly lower in the diabetic group than in the nondiabetic group $(P<0.03)$ and lower in the PDR group than in the nondiabetic and NPDR groups $(P<0.01$ and $P<0.03$, respectively), but no significant difference was found between the nondiabetic and NPDR groups. The daytime melatonin level did not significantly differ between the nondiabetic and diabetic groups or between the nondiabetic, NPDR, and PDR groups.

Conclusion: The nighttime melatonin level is altered in patients with diabetes and PDR but not in diabetic patients without PDR. Although patients with PDR may have various dysfunctions that affect melatonin secretion more severely, advanced dysfunction of retinal light perception may cause altered melatonin secretion. Alteration of melatonin secretion may accelerate further occurrence of complications in diabetic patients.

Keywords: circadian rhythm, diabetes, proliferative diabetic retinopathy, melatonin

\section{Introduction}

Melatonin, an indoleamine hormone, is synthesized by pinealocytes, retinal photoreceptors, and ciliary epithelial cells. ${ }^{1,2}$ The pineal gland secretes melatonin into the circulation almost entirely at night in vertebrates. ${ }^{2,3}$ The retinal perception of environmental light and dark cycles is transmitted to the suprachiasmatic nucleus via the retinohypothalamic tract. Inhibitory information carried by the axons from the suprachiasmatic nucleus is transferred to the autonomic division of the paraventricular nucleus and the brainstem areas involved in sympathetic preganglionic outflow. The axons of these neurons are connected to the intermediolateral cell column of the spinal cord, which carries input to the superior cervical ganglia, which is the primary source of pineal innervation. ${ }^{4,5}$ This complex pathway of environmental light/dark signal transduction is important for the circadian rhythmicity of melatonin secretion.

Melatonin physiology in diabetes mellitus has been examined. Animal and human studies have documented decreased plasma melatonin levels in diabetes. ${ }^{6-12}$ Chronic complications of diabetes, especially retinopathy and autonomic neuropathy, would be expected to affect nocturnal melatonin secretion. Ha et $\mathrm{al}^{13}$ investigated the effect 
of melatonin on signaling pathways for glucose transport in mouse skeletal muscle cells, and demonstrated that melatonin stimulated glucose transport to skeletal muscle cells via the IRS-1/PI-3 kinase pathway, which implies, at the molecular level, its role in glucose homeostasis and possibly in diabetes. Additionally, exposure to light at night and aging, both of which lower endogenous melatonin levels, may contribute to the incidence and/or development of diabetes. Furthermore, Contreras-Alcantara et al ${ }^{14}$ have demonstrated recently that removal of the type 1 melatonin receptor (MT1) significantly impairs the ability of mice to metabolize glucose, probably due to increased insulin resistance, and suggesting that MT1 receptors are implicated in the pathogenesis of type 2 diabetes. This research paved the way for detailed exploration of the mechanisms by which MT1 receptor signaling might affect glucose metabolism. However, we did not find any publications on altered melatonin levels in diabetic patients with and without retinopathy in the scientific literature. In the present study, we examined plasma melatonin secretion in patients with type 2 diabetes and diabetic retinopathy.

\section{Materials and methods Subjects}

Fifty-six patients hospitalized at Ohtsuka Eye Hospital to undergo eye surgery participated in this study. Written informed consent was obtained from all patients, and the study adhered to the tenets of the Declaration of Helsinki. The 56 patients were divided into two groups, ie, a diabetic group comprising 30 patients with type 2 diabetes, and a nondiabetic group, comprising 26 patients whose biochemical and physiologic parameters (serum glucose, complete blood and platelet counts, liver enzymes, serum creatinine level, hemoglobin A1c [HbAlc], dipstick urine analysis, and electrocardiography) were within normal limits, and with no history of cardiac events. The diabetic group was further divided into patients with proliferative diabetic retinopathy (PDR, $n=14$ ) and nonproliferative diabetic retinopathy (NPDR, $n=16)$, including those with no retinopathy and mild NPDR. Retinopathy was documented during a standard fundus examination in all patients, who underwent fluorescein angiography if necessary to evaluate the presence of retinal neovascularization. Exclusion criteria included cigarette smoking, sleep disorders (including obstructive sleep apnea), systemic disease or cardiac arrhythmia, and concomitant drugs affecting the autonomic nervous system, including $\alpha$ - and $\beta$-adrenergic receptor blockers and other sympatholytic agents, antidepressants, and anorectics. Renal function was assessed by serum levels of blood urea nitrogen and creatinine and by measuring for microalbuminuria. Peripheral polyneuropathy was evaluated by the same experienced endocrinologist, and was defined by the presence of symptoms, including pain, numbness, and paresthesia, and the absence of ankle reflexes.

Patients in the nondiabetic group and the NPDR group were admitted to hospital to undergo cataract surgery and those in the PDR group were admitted to undergo vitreous surgery combined with cataract surgery for massive vitreous hemorrhage, tractional retinal detachment, or both, associated with PDR. The lights in the hospital were turned off at $9 \mathrm{pm}$ and turned on at 6 am.

Circadian fluctuations in melatonin were assessed by drawing blood in dim light (0 lux) via an indwelling cannula from an antecubital vein for measurement of melatonin levels at midnight and 12.30 am while the patients were sleeping, and in the afternoon between $3 \mathrm{pm}$ and $3.30 \mathrm{pm}$. Particular attention was paid to allowing the participants to remain asleep during the midnight blood collection. Blood samples were obtained from all patients 2 days after surgery. Plasma melatonin was measured by high-performance liquid chromatography in all patients. This method has proven reproducibility and can detect a $0.5 \mathrm{pg} / \mathrm{mL}$ plasma concentration of melatonin, which is a lower value than that of the lowest circadian level of melatonin. Our preliminary study showed that the plasma concentration of melatonin obtained from five nondiabetic volunteers peaked at around midnight and was lowest at around 3 pm (Figure 1).

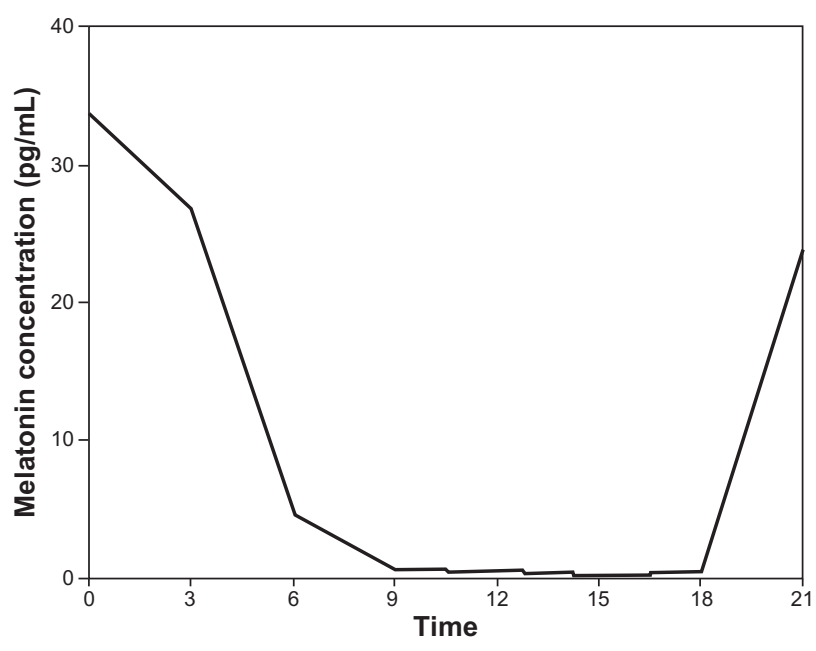

Figure I Typical circadian fluctuations in plasma melatonin from five nondiabetic volunteers. The plasma concentration of melatonin reached its highest level at around midnight and lowest at around $3 \mathrm{pm}$. Vertical lines are one standard error of the means. 


\section{Statistical analysis}

The differences between the patient groups were assessed for statistical significance using Student's $t$-test or repeatedmeasures analysis of variance as appropriate. The results are expressed as the mean \pm standard deviation unless otherwise indicated. Analysis was conducted using the SPSSfor Windows software package (v 11.5.1; SPSS Inc, Chicago, IL). $P<0.05$ was considered statistically significant.

\section{Results}

The clinical and laboratory characteristics of the patients are shown in Table 1. No significant differences were found for age or gender between the groups. All women in this study were postmenopausal and not receiving hormone therapy. All patients in the PDR group had undergone bilateral panretinal photocoagulation.

The minimum angle of resolution (log MAR) visual acuities converted from patients' Randolt rings showed that best-corrected visual acuities for the better and worse eyes were significantly better in the nondiabetic group than in the diabetic group $(P<0.01$, respectively, Student's $t$-test $)$ and the PDR group $(P<0.01$, respectively, repeated-measures analysis of variance), whereas no significant difference was found between the nondiabetic and NPDR groups (Table 1). In the PDR group, the logMAR visual acuity of the worse eye was significantly lower compared with the NPDR group $(P<0.01$, repeated-measures analysis of variance), but the $\log$ MAR visual acuities of the better eye did not differ significantly between the PDR and NPDR groups.

In the diabetic group, the percentages of patients who used insulin or had diabetic neuropathy and peripheral polyneuropathy did not differ significantly between the NPDR and PDR groups. There was no significant difference in $\mathrm{HbAlc}$ between the NPDR and PDR groups (Table 1).

The nighttime and daytime melatonin levels were $35.5 \pm 33.4 \mathrm{pg} / \mathrm{mL}$ and $2.8 \pm 3.0 \mathrm{pg} / \mathrm{mL}$ in the nondiabetic group, $23.6 \pm 30.1$ and $1.0 \pm 0.9 \mathrm{pg} / \mathrm{mL}$ in the diabetic group, $34.8 \pm 37.4$ and $1.33 \pm 0.7 \mathrm{pg} / \mathrm{mL}$ in the NPDR group, and $10.9 \pm 11.4$ and $0.7 \pm 0.9 \mathrm{pg} / \mathrm{mL}$ in the PDR group (Figures 2 and 3). The nighttime melatonin level was significantly lower in the diabetic group than in the nondiabetic group $(P<0.03$, Student's $t$-test $)$ and in the PDR group than in the nondiabetic and NPDR groups $(P<0.01$ and $P<0.03$, repeated-measures analysis of variance), but no significant

Table I Clinical and laboratory characteristics of each group of study patients

\begin{tabular}{|c|c|c|c|c|}
\hline & \multicolumn{3}{|l|}{ Diabetic } & \multirow[t]{2}{*}{ Nondiabetic } \\
\hline & & NPDR & PDR & \\
\hline Patients (n) & 30 & 16 & 14 & 26 \\
\hline Age (years) & $65 \pm 10$ & $72 \pm 8$ & $56 \pm 12$ & $66 \pm 11$ \\
\hline Gender (M/F) & $11 / 19$ & $5 / 11$ & $6 / 8$ & $10 / 16$ \\
\hline Duration (years) & $12 \pm 6$ & $14 \pm 7$ & $10 \pm 6$ & - \\
\hline \multicolumn{5}{|l|}{ from diagnosis of DM } \\
\hline HbAlc (\%) & $6.8 \pm 2.4$ & $7.1 \pm 3.2$ & $6.4 \pm 1.3$ & - \\
\hline \multicolumn{5}{|l|}{ Diabetes treatment } \\
\hline Diet only & I (3\%) & I (6\%) & $0(0 \%)$ & - \\
\hline Oral drug & $23(77 \%)$ & $12(75 \%)$ & II (79\%) & - \\
\hline Insulin & $6(20 \%)$ & $3(19 \%)$ & $3(2 \mid \%)$ & - \\
\hline \multicolumn{5}{|l|}{ Increased BUN } \\
\hline And/or creatinine & 7 (23\%) & $2(13 \%)$ & $5(36 \%)$ & - \\
\hline Microalbuminuria & 8 (27\%) & $3(19 \%)$ & $5(36 \%)$ & - \\
\hline Distal polyneuropathy & $9(30 \%)$ & $3(19 \%)$ & $6(43 \%)$ & - \\
\hline Body mass index & $27.5 \pm 3.2$ & $28.1 \pm 3.5$ & $27.0 \pm 3.0$ & $24.6 \pm 3.4$ \\
\hline \multicolumn{5}{|l|}{ Log MAR VA } \\
\hline Better eye & $0.30 \pm 0.32$ & $0.13 \pm 0.10$ & $0.51 \pm 0.36$ & $0.11 \pm 0.09$ \\
\hline Worse eye & $0.88 \pm 0.70$ & $0.31 \pm 0.20$ & $1.53 \pm 0.40$ & $0.27 \pm 0.15$ \\
\hline \multicolumn{5}{|l|}{ Mean decimal VA* } \\
\hline Better eye & 0.50 & 0.75 & 0.30 & 0.79 \\
\hline Worse eye & 0.14 & 0.49 & 0.03 & 0.53 \\
\hline \multicolumn{5}{|l|}{ Melatonin } \\
\hline Nighttime (pg/mL) & $20.4 \pm 22.3$ & $31.1 \pm 26.5$ & $10.9 \pm 11.4$ & $37.5 \pm 30.8$ \\
\hline Daytime (pg/mL) & $0.8 \pm 0.7$ & $\mathrm{I} . \mathrm{I} \pm 0.6$ & $0.4 \pm 0.7$ & $2.4 \pm 4.1$ \\
\hline
\end{tabular}

Note: *Mean decimal VA was converted from mean logMAR VA.

Abbreviations: BUN, blood urea nitrogen; DM, diabetes; NPDR, nonproliferative diabetic retinopathy; HbAIc, glycosylated hemoglobin; VA, visual acuity; Log MAR, minimum angle of resolution; PDR, proliferative diabetic retinopathy. 


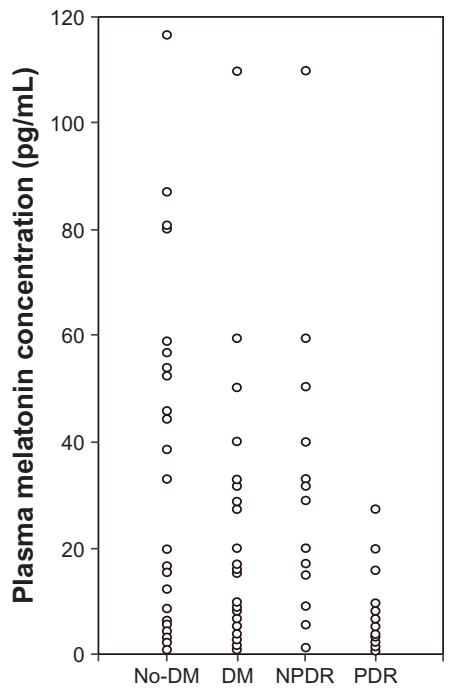

Figure 2 Nighttime melatonin levels in each group. The nighttime melatonin level differed significantly between the nondiabetic $(n=26)$ and diabetic groups $(n=30$, $P<0.03$, Student's $t$-test), and that level was significantly lower in the proliferative diabetic retinopathy group $(n=14)$ than in the nondiabetic and nonproliferative diabetic retinopathy groups $(n=16, P<0.01$ and $P<0.03$, repeated-measures analysis of variance), but no significant difference was found between the nondiabetic and nonproliferative diabetic retinopathy groups.

difference was found between the nondiabetic and NPDR groups. The daytime melatonin level did not significantly differ between the nondiabetic and diabetic groups, or between the nondiabetic, NPDR, and PDR groups.

\section{Discussion}

To the best of our knowledge, this is the first study to evaluate the relationship between melatonin levels and diabetic retinopathy. Our results show that alteration of nighttime melatonin level was more apparent in patients with PDR.

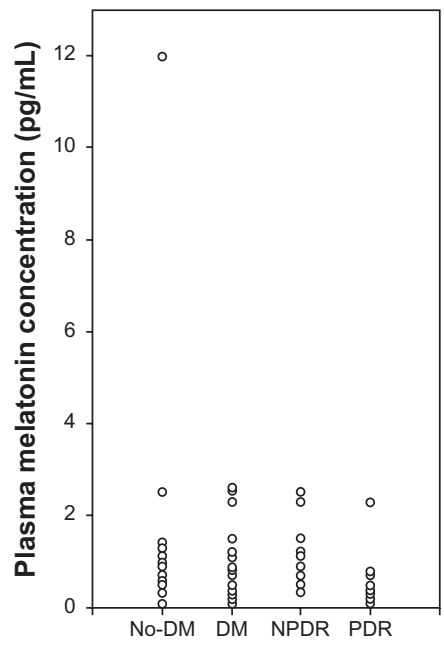

Figure 3 Daytime melatonin levels in each group. The daytime melatonin level did not significantly differ between the nondiabetic $(n=26)$ and diabetic groups $(n=30)$ or among the nondiabetic, nonproliferative diabetic retinopathy $(n=16)$, and proliferative diabetic retinopathy $(n=14)$ groups.
Experimental diabetes resulted in decreased synthesis of melatonin from pinealocytes and decreased activity of hydroxyindole-O-methyltransferase, which is a key enzyme in melatonin synthesis. ${ }^{8,9}$ Recent publications confirm a functional interrelationship between melatonin and insulin, and a reduction of melatonin in the genesis of diabetes. ${ }^{11,12,15-18}$

Although Tutuncu et $\mathrm{a}^{10}$ reported that the circadian rhythm of melatonin secretion was deteriorated in patients with type 2 diabetes and that there was a complex relationship between various components of the autonomic nervous system and nighttime melatonin secretion, they did not find a difference in the melatonin levels of diabetic patients with and without retinopathy, and suggested that a small number of patients with PDR but without advanced retinopathy might have masked the effect of diabetic retinopathy on melatonin physiology in their study. The retinal perception of environmental light and dark cycles is transmitted to the autonomic nerve system and finally to the pineal gland. Thus, disordered melatonin secretion induced by autonomic neuropathy seems reasonable.

Shanahan et a ${ }^{19}$ emphasized the importance of considering both phase and amplitude when describing the resetting of the human circadian pacemaker such as melatonin and temperature rhythms by light. Output signals from the suprachiasmatic nucleus regulate mRNA levels of pineal arylalkylamine $\mathrm{N}$-acetyltransferase, a key enzyme in the melatonin synthesis pathway. Nocturnal upregulation of arylalkylamine $\mathrm{N}$-acetyltransferase mRNA is the main cause for increased melatonin synthesis at night. ${ }^{20,21}$ Light is the most prevalent environmental stimulus that synchronizes the circadian pacemaker at the suprachiasmatic nucleus. In addition, light exerts direct effects on many of the pacemaker's rhythmic outputs, including melatonin release. Schwartz et a ${ }^{22}$ showed that entrainment of the circadian rhythm of melatonin release could indeed be dissociated from its inhibition by light.

In the current study, visual acuities in both eyes were significantly worse in the PDR groups, which may indicate dysfunctional retinal light perception. Moreover, all patients in the PDR group had undergone bilateral panretinal photocoagulation. Although panretinal photocoagulation is an established treatment to prevent retinal neovascularization, ${ }^{23}$ the photocoagulated retinal and choroidal lesions are damaged anatomically and functionally. ${ }^{24}$ Retinal dysfunction induced not only by advanced diabetic retinopathy but also by panretinal photocoagulation should be associated with diminished retinal light perception. Bughi et $\mathrm{al}^{25}$ reported that bilateral photocoagulation for PDR may be associated with loss of normal circadian cortisol variation. Therefore, not 
only severe diabetic retinopathy may be contributing to the decreased nighttime melatonin level, but also a significantly decreased area of functioning rods in lasered retinas. Lockley et $\mathrm{al}^{26}$ reported that most blind people without light perception had a circadian rhythm disorder. On the contrary, all patients without PDR in the current study were planning to undergo cataract surgery to improve visual acuity, ie, visual disturbance was considered to be the result of cataract and nondiabetic retinopathy in these patients. Thus, the degree of visual dysfunction induced by diabetic retinopathy should have been mild in these patients. Moreover, visual acuities of the better eyes were superior to those of PDR patients and visual acuities of the worse eyes, and these findings were similar to those in nondiabetic patients. Thus, cataracts in patients without PDR did not disrupt photic transmission to the retina and suprachiasmatic nucleus.

This preliminary study provides data showing disturbed melatonin secretion in patients with PDR. We also speculated that if advanced bilateral dysfunction of retinal light perception alters melatonin secretion, patients with decreased visual acuities in both eyes resulting from other disorders may also have dysfunctional melatonin secretion. Although panretinal photocoagulation, which is an essential treatment for PDR, may overestimate disturbed melatonin secretion in patients with PDR, advanced diabetic retinopathy seems to be an important factor for decreased melatonin secretion.

Limitations of this study were its small sample size and the standard deviations of the mean plasma concentrations of melatonin being large. Because plasma melatonin concentrations were measured at only two time points during 24 hours in each subject, we could not demonstrate the entire rhythm of melatonin secretion over 24 hours in each subject. However, it is unclear as to what phase of the rhythm this midnight sample refers to because it is difficult to know the typical sleep times for each subject in the current study, and frequent drawing of blood during sleeping time in the hospital would not be allowed by the institutional review board at Ohtsuka Eye Hospital. Further and more detailed studies in a large number of diabetic participants with various diabetic complications are needed to determine their melatonin depletion status and its consequences in terms of biorhythm imbalances.

\section{Disclosure}

The authors report no conflicts of interest in this work.

\section{References}

1. Lerner AB, Case JD, Takahashi Y. Isolation of melatonin and 5-methoxyindole-3-acetic acid from bovine pineal glands. J Biol Chem. 1960;235(7):1992-1997.
2. Cahill GM, Besharse JC. Light-sensitive melatonin synthesis by Xenopus photoreceptors after destruction of the inner retina. Vis Neurosci. 1992;8(5):487-490.

3. Cahill GM, Grace MS, Besharse JC. Rhythmic regulation of retinal melatonin: Metabolic pathways, neurochemical mechanisms, and the ocular circadian clock. Cell Mol Neurobiol. 1991;11(5): 529-560.

4. Tan DX, Manchester LC, Terron MP, Flores LJ, Reiter RJ. One molecule, many derivatives: A never-ending interaction of melatonin with reactive oxygen and nitrogen species? J Pineal Res. 2007;42(1):28-42.

5. Simko F, Paulis L. Melatonin as a potential antihypertensive treatment. J Pineal Res. 2007;42(4):319-322.

6. O'Brien IA, Lewin IG, O'Hare JP, Arendt J, Corrall RJ. Abnormal circadian rhythm of melatonin in diabetic autonomic neuropathy. Clin Endocrinol (Oxf). 1986;24(4):359-364.

7. Nielsen FS, Hansen HP, Jacobsen P, et al. Increased sympathetic activity during sleep and nocturnal hypertension in type 2 diabetic patients with diabetic nephropathy. Diabet Med. 1999; 16(7):555-562.

8. Champney TH, Brainard GC, Richardson BA, Reiter RJ. Experimentallyinduced diabetes reduces nocturnal pineal melatonin content in the Syrian hamster. Comp Biochem Physiol. 1983;76(1):199-201.

9. Pang SF, Tang F, Tang PL. Alloxan induced diabetes and the pineal gland: Differential effects on the levels of pineal N-acetyl serotonin, pineal melatonin, and serum melatonin. J Pineal Res. 1985;2(1): 79-85.

10. Tutuncu NB, Batur MK, Yildirir A, et al. Melatonin levels decrease in type 2 diabetic patients with cardiac autonomic neuropathy. $J$ Pineal Res. 2005;39(1):43-49.

11. Peschke E, Frese T, Chankiewitz E, et al. Diabetic Goto Kakizaki rats as well as type 2 diabetic patients show a decreased diurnal serum melatonin level and an increased pancreatic melatonin-receptor status. J Pineal Res. 2006;40(2):135-143.

12. Nishida S, Sato R, Murai I, Nakagawa S. Effect of pinealectomy on plasma levels of insulin and leptin and on hepatic lipids in type 2 diabetic rats. J Pineal Res. 2003;35(4):251-256.

13. Ha E, Yim S, Chung J, et al. Melatonin stimulates glucose transport via insulin receptor substrate-1/phosphatidylinositol 3-kinase pathway in C2C12 murine skeletal muscle cells. J Pineal Res. 2006;41(1):67-72.

14. Contreas-Alcantara S, Baba K, Tosini G. Removal of melatonin receptor type 1 induced insulin resistance in the mouse. Obesity. 2010; 18(9):1861-1863.

15. Nishida S, Segawa T, Murai I, Nakagawa S. Long-term melatonin administration reduces hyperinsulinemia and improves the altered fatty-acid compositions in type 2 diabetic rats via the restorations of Delta-5 desaturase activity. J Pineal Res. 2002;32(2):26-33.

16. Kanter M, Uysal H, Karaca T, Sagmanligil HO. Depression of glucose levels and partial restoration of pancreatic beta-cell damage by melatonin in streptozotocin-induced diabetic rats. Arch Toxicol. 2006; 80(6):362-369.

17. Peschke E, Stumpf I, Bazwinsky I, Litvak L, Dralle H, Muhlbauer E. Melatonin and type 2 diabetes - a possible link? J Pineal Res. 2007;42(4): $350-358$.

18. Stebelova K, Herichova I, Zeman M. Diabetes induced changes in melatonin concentrations in peripheral tissues of rat. Neuro Endocrinol Lett. 2007;28(2):159-165.

19. Shanahan TL, Kronauer RE, Duffy JF, Williams GH, Czeisler CA. Melatonin rhythm observed throughout a three-cycle bright-light stimulus designed to reset the human circadian pacemaker. J Biol Rhythms. 1999;14(3):237-253.

20. Borjigin J, Wang MM, Snyder SH. Diurnal variation in mRNA encoding serotonin $\mathrm{N}$-acetyltransferase in pineal gland. Nature. 1995;378(6559): $783-785$.

21. Roseboom PH, Coon SL, Baler R, McCune SK, Weller JL, Klein DC. Melatonin synthesis: Analysis of the more than 150-fold nocturnal increase in serotonin $\mathrm{N}$-acetyltransferase messenger ribonucleic acid in the rat pineal gland. Endocrinology. 1996;137(7):3033-3045. 
22. Schwartz MD, Wotus C, Liu T, et al. Dissociation of circadian and light inhibition of melatonin release through forced desynchronization in the rat. Proc Natl Acad Sci U S A. 2009;106(41):17540-17545.

23. Diabetic Retinopathy Study Research Group. Preliminary report on effects of photocoagulation therapy. Am J Ophthalmol. 1976;81(4): 383-396.

24. Perlam I, Gdal-On M, Miller B, Zonis S. Retinal function of the diabetic retina after argon laser photocoagulation assessed electroretinographically. Br J Ophthalmol. 1985;69(4):240-246.
25. Bughi S, Shaw S, Bessman A. Laser damage to retinal ganglion cells: The effect on circadian rhythms. J Diabetes Complications. 2006;20(3):184-187.

26. Lockley SW, Arendt J, Skene DJ. Visual impairment and circadian rhythm disorders. Dialogues Clin Neurosci. 2007;9(3):301-314.

\section{Publish your work in this journal}

Clinical Ophthalmology is an international, peer-reviewed journal covering all subspecialties within ophthalmology. Key topics include: Optometry; Visual science; Pharmacology and drug therapy in eye diseases; Basic Sciences; Primary and Secondary eye care; Patient Safety and Quality of Care Improvements. This journal is indexed on

\section{Dovepress}

PubMed Central and CAS, and is the official journal of The Society of Clinical Ophthalmology (SCO). The manuscript management system is completely online and includes a very quick and fair peer-review system, which is all easy to use. Visit http://www.dovepress.com/ testimonials.php to read real quotes from published authors. 